\title{
LOS ESTUDIANTES UNIVERSITARIOS ANTE LA INCLUSIÓN DE SUS COMPAÑEROS CON DISCAPACIDAD: INDICADORES BASADOS EN LA TEORÍA DE LA ACCIÓN RAZONADA PARA LOS ESTUDIOS DE ECONOMÍA Y EMPRESA EN LA UNIVERSIDAD DE A CORUÑA (ESPAÑA)
}

\author{
UNIVERSITY STUDENTS FACING INCLUSION OF THEIR COLLEGUES WITH \\ DISABILITIES: INDICATORS BASED ON THE THEORY OF PLANNED BEHAVIOR \\ FOR ECONOMICS AND BUSINESS STUDIES IN THE UNIVERSITY OF A CORUÑA
} (SPAIN)

\author{
Isabel Novo Corti \\ Jesús Miguel Muñoz Cantero ${ }^{1}$ \\ Universidad de A Coruña
}

\section{RESUMEN}

El planteamiento de la inclusión en la educación ordinaria o tradicional de los colectivos con diversidad funcional o con discapacidad, ha sido ampliamente reconocido como el camino más adecuado para la consecución de los mejores resultados no sólo académicos sino también de bienestar social y económico. A pesar de que se han hecho grandes avances en los niveles educativos primario y secundario, la educación superior ha estado en muchas ocasiones vetada para estos colectivos, quienes han tenido que recurrir a las Universidades a distancia como única opción. En este trabajo, se analiza la inclusión de los colectivos con discapacidad en el ámbito de la Universidad tradicional, presencial, haciendo hincapié en la importancia de la actitud del colectivo estudiantil, en su conjunto, para que dicha inclusión sea exitosa. Planteamos un trabajo

\footnotetext{
${ }^{1}$ Correspondencia: Jesús Miguel Muñoz Cantero: Facultad de Ciencias de la Educación. Departamento de Filosofía y Métodos de Investigación en Educación. Campus de Elviña s/n A Coruña 15071. Correo-e: jesus.miguel.munoz@udc.es
} 
fundamentado en una investigación empírica realizada en la Facultad de Economía y Empresa de la Universidad de A Coruña, durante el año 2010, a través de una encuesta a 164 estudiantes basada en el estudio de las actitudes, normas sociales y control percibido, como determinantes de la intención de apoyar a la inclusión, siguiendo la Teoría del comportamiento planeado (TPB). Los resultados alcanzados son esperanzadores, sin embargo muestran la necesidad de que las instituciones públicas promuevan políticas educativas inclusivas en las Universidades. Se confirma que la convivencia es determinante para mejorar las actitudes hacia la discapacidad.

Palabras clave: Educación Superior, estudiantes con discapacidad, actitudes, discapacidad, educación Inclusiva.

\section{ABSTRACT}

Inclusive education for disabled people has been broadly accepted like the best way to achieve the best results, both in relation to academic success and in boosting social integration and social and economic welfare. Although great progress have been made in primary and secondary education, access to higher education has been almost impossible too often for many people in these groups, for whom distance university has been their only option until recently. In our work, we have intended to analyze the inclusion of disabled people in a conventional university environment, with face to face teaching, focusing on the attitudes of all students, as a group, and the great importance of their behavior to achieve a successful inclusion. We propose a work based on an empirical investigation undertaken in the Faculty of Economics and Business of the University of A Coruña (Spain). A total of 164 students were enquired on individual attitudes, social norms and perceived control as key questions for promoting intention to support inclusion of disabled students, as the Theory of Planned Behavior postulates (TPB). The achieved results are encouraging, nevertheless they show the need of public institutions as boosters of educational inclusive policies at the Universities. Coexistence is confirmed as a key variable to promote positive attitudes towards disability.

Key words: High Education, disabled students, attitudes, disability, inclusive education.

\section{Introducción}

La influencia de los niveles educativos en la calidad de vida y el bienestar alcanzado por las sociedades se ha puesto reiteradamente de manifiesto. El estado del bienestar ha representado una prioridad para los gobiernos y sociedades desarrolladas, de ahí la importancia de la elaboración de políticas educativas que promuevan la consecución de estos objetivos. Sin embargo, a pesar de ello, todavía existen numerosos grupos o colectivos en riesgo de exclusión social tales como inmigrantes, personas con discapacidad, pobres, familias monoparentales, etnias minoritarias, personas en edad avanzada, etc., cuyo riesgo de exclusión se amplía también a la esfera universitaria. En este trabajo planteamos la inclusión de las personas con discapacidad en la universidad presencial.

En relación con la inclusión en contextos educativos básicos existen numerosos estudios, algunos de los cuales muestran resultados claramente optimistas como el de Horrocks, White y 
Roberts $(2008)^{2}$ o las propuestas de Palacios (2010) o Dueñas Buey (2010) en las que ensalza el modelo social de discapacidad sobre el modelo de deficiencia. Las actitudes del entorno, en sentido amplio, han sido consideradas clave sustancial para la inclusión educativa. En este sentido apuntan los trabajos de Murray (2011) y Rodríguez-Ascaso (2011) que ponen el acento en la importancia de factores extra-académicos, como por ejemplo el staff del centro educativo o la accesibilidad, respectivamente.

Es patente la relativa escasez de trabajos de investigación enfocados hacia la inclusión en contextos educativos superiores, posiblemente debido a la dificultad que el colectivo de las personas con discapacidad tiene para acceder a dichos niveles. Uno de los escasos trabajos sobre este particular es el realizado por Collins et al (2009), en la Universidad de Kentuky, sobre la inclusión en la educación superior a través de la enseñanza a distancia. Es precisamente la enseñanza a distancia la que se ha manifestado en muchas ocasiones como una opción que permite a las personas con discapacidad alcanzar niveles de formación universitaria.

\section{Los conceptos de discapacidad y riesgo de exclusión}

\section{Discapacidad}

Este concepto se mueve en un terreno complejo donde confluyen multiplicidad de términos vinculados a concepciones médicas, sociales, psicológicas o educativas (Verdugo, 1995; Dueñas Buey, 2010; Palacios, 2008). Los primeros estudios en torno a este tema comenzaron en los años 60 en los Estados Unidos entre los que destacan las investigaciones de Haber (1967), Nagi (1979) y Altman (1986). Durante décadas posteriores Bickenbach et al. (1999), Fujiura y Rutkowski Kmitta (2001), Gross \& Hahn (2004), Hahn \& Pool Hegamin (2001), Grönvik (2009) han puesto de manifiesto que los investigadores han luchado para encontrar definiciones operativas de discapacidad que sean «completas», «globales» o «estables en el tiempo», pero el problema de la discapacidad como un concepto, al igual que ocurre con muchos otros conceptos en las ciencias sociales, radica en sus circunstancias (Aspinall, 1997).

Tras la revisión de distintas propuestas sobre la terminología de la discapacidad (Grönvik, 2009; Szebehely, Fritzell, y Lundberg, 2001; Martin, Meltzer y Elliot, 1988; Naciones Unidas, 1990, 1996 y 2003; Barnes y Mercer, 2004; Shakespeare, 2005). En este trabajo, nos referimos a un concepto de discapacidad centrado en los aspectos sociales, lo que se denomina "diversidad funcional" de acuerdo a Alonso et al (2007) entendiendo la discapacidad en sentido amplio, como aquella dificultad especial que alguna persona tiene en un área concreta, sea física, sensorial o psicológica.

\section{El riesgo de exclusión social}

El concepto de exclusión social se atribuye a René Lenoir (1974). Bajo el paraguas de este concepto se cobijaba una amplia variedad de personas: no sólo los pobres, sino también las personas con discapacidad, los suicidas y personas mayores, los chicos que habían sufrido abusos, etc. Más adelante, este término fue utilizado para referirse a varios tipos de desventajas sociales, relacionados con los nuevos problemas sociales que iban apareciendo vinculados a las

\footnotetext{
${ }^{2}$ Han estudiado la actitud hacia la inclusión de niños con autismo en contextos educativos normalizados de escuelas públicas de Pensilvania (Estados Unidos), llegando a la conclusión de que esta inclusión podría articularse sin generar especiales dificultades.
} 
situaciones de crisis: desempleo, marginación y cambios fundamentales en la vida de las familias (Cannan \& Warren 1997). En trabajos como el de Prescott (2011) se ha comprobado cómo la mejora en los estándares de salud permite un mayor acceso a la educación superior de ciertos colectivos en riesgo de exclusión, como personas con discapacidad, mujeres, etc. En el terreno internacional, el Programa de Desarrollo de las Naciones Unidas (PNUD) ha estado a la vanguardia de los intentos de conceptualizar la exclusión social en los países desarrollados y en desarrollo. Si además pretendemos avanzar en el modelo de Bienestar Social y calidad de vida, la educación superior debe formar parte de los niveles educacionales accesibles. Este es el concepto en el que nos vamos a fundamentar.

\section{Factores que influyen en la predisposición a apoyar a los estudiantes universitarios con discapacidad por parte de su compañeros}

Existen factores claves determinantes de la propensión a la inclusión que se pueden clasificar siguiendo la Teoría del Comportamiento Planeado -Theory of Planned Behaviour (TPB)- (Ajzen, 1991) que investiga, además de la actitud del agente involucrado, en este caso el estudiante universitario, otras variables como la norma social y el control percibido. Por un lado, está la norma social o influencia de los familiares, amigos o terceras personas en el comportamiento de los agentes y, por otro, el control percibido que recoge las habilidades, capacidades, destrezas que influirán positivamente en la intención del agente en variables actitudinales, sociales y de autopercepción. Todos estos aspectos podrían ser explicativos de la intención inclusiva de los estudiantes universitarios, esta es pues la cuarta variable que hemos tomado en consideración.

Entendemos por actitud (Fishbein y Ajzen, 1974) la predisposición del agente hacia las personas con discapacidad. Esta predisposición puede ser cognitiva, afectiva o comportamental. Estas opiniones, sentimientos e intenciones hacia la persona con discapacidad podrían influir de forma muy positiva en la integración e inclusión social. Asimismo, una actitud positiva de los agentes sería la base para generar entornos inclusivos favorables que auspicien un cambio de mentalidad hacia estos colectivos (Clore y Jeffrey, 2007). Los numerosos estudios acometidos sobre este particular en enseñanzas no universitarias (por citar algunos de los más recientes, los de Sharma, et al 2009, Ernst y Rogers 2009) alcanzan resultados dispares, si bien en todos ellos se encuentra una mayor relación entre los niveles de formación -tanto de profesorado como de alumnado- y propensión a actitudes inclusivas. Referidos a estudiantes universitarios/as Infante y Gómez (2004) han examinado la actitud inclusiva hacia la discapacidad como la multiculturalidad de los/as estudiantes universitarios/as, según distintas áreas de conocimiento y de distintos niveles; o el de Alonso et al (2007) que analizan las actitudes de los estudiantes universitarios hacia los colectivos en riesgo de exclusión (lo que ellos denominan "diferencias"). En relación con la actitud favorable a la inclusión por parte del alumnado universitario están los trabajos de Sánchez Bravo et al (2008) para alumnos de Pedagogía; o el trabajo de Luque y Rodríguez (2008) sobre los elementos a tener en cuenta con alumnado universitario con discapacidad. Más recientemente podemos citar el trabajo de Surriá Martínez (2011) que realiza un análisis de actitudes hacia la discapacidad en la Universidad de Alicante.

Los problemas de exclusión social de las personas con discapacidad y de otros colectivos en riesgo de exclusión son mirados con distintas lentes según las sociedades. Por este motivo cabe esperar que las actitudes de los jóvenes hacia la inclusión sean diferentes en función del entorno en el que se encuentren. De ahí la importancia de lo que hemos denominado "Norma Social". Se trata de la percepción que tiene el agente de la influencia que ejercen terceras personas o grupos 
específicos relevantes cuando decide realizar una determinada acción y que hacen que el agente actúe en una dirección determinada, guiada por esta fuerza externa. En ella destacamos la influencia de amigos, familiares, rol de las instituciones educativas, etc. Como reflejo de este sentir social, la Unión Europea se ha comprometido en la lucha contra la exclusión social, que se ha manifestado en los tratados de Maastricht y de Amsterdam y en los Fondos Sociales Europeos que se han implicado fuertemente en combatir la exclusión social. Las actuaciones más recientes en la Unión Europea surgen a raíz de la comunicación de la Comisión "Europa 2020".

Pero además de estos dos factores, existe un tercero, de gran importancia, basado en las capacidades, destrezas y habilidades percibidas por los agentes y que hay que tener para prestar ayuda a una persona con cierto grado de discapacidad. Los estudios de control percibido por parte de los jóvenes, se dirigen hacia diversos campos que son objeto especial de atención en estas edades, algunos se relacionan con la salud: McCaul et al (1993), otros con seguridad: White et al (2008), Nemme et al (2010), los usos de internet: Pelling y White (2009), etc. Se han realizado también estudios sobre el control percibido por las personas con discapacidad, como los de Paul et al (2006), Lannen et al (2002) sobre jóvenes con dificultades de aprendizaje en entornos virtuales y el de Mueller et al (2006) sobre jóvenes con Síndrome de Tourette, en el contexto universitario.

Por otra parte, hemos considerado la intención a ayudar a los estudiantes con discapacidad como una variable relevante, que en gran medida podría venir explicado por los tres constructos anteriores. Los cuatro aspectos descritos en los párrafos anteriores son los que hemos tenido en cuenta para la elaboración del análisis descriptivo de la situación actual en el contexto de la enseñanza de titulaciones de Economía y Empresa en la Universidad de A Coruña (España), como un estudio de caso, cuya metodología explicamos a continuación

Tomando en cuenta lo anterior, las preguntas a las que pretendemos dar respuesta son las siguientes:

- Pregunta 1: ¿Las actitudes hacia las personas con discapacidad influyen sobre la intención apoyo a la inclusión de los estudiantes universitarios que la padecen?.

- Pregunta 2: ¿Cuanto más favorable sea la norma social dominante, mayor será la intención de apoyo a la inclusión de los estudiantes con discapacidad, por parte de sus compañeros?.

- Pregunta 4: ¿Cuanto mayor sea el control percibido por los estudiantes universitarios sobre su capacidad de ayuda a las personas con discapacidad, mayor será la intención de apoyo a la inclusión de los compañeros que la sufren?.

- Pregunta 4: ¿La intención de apoyar a la inclusión de los estudiantes universitarios con discapacidad, por parte de sus compañeros, responde a los supuestos de la Teoría del Comportamiento Planeado?.

- Pregunta 5: ¿Existen diferencias entre hombres y mujeres en los aspectos estudiados en este trabajo (actitudes, norma social y control percibidos, e intención de apoyo a la inclusión)?

- Pregunta 6: ¿Existen diferencias entre las personas que conviven con personas con discapacidad en los aspectos estudiados en este trabajo (actitudes, norma social y control percibidos, e intención de apoyo a la inclusión)?

De este modo las hipótesis nulas o hipótesis a contrastar son, respectivamente, las siguientes:

- H01: La intención de apoyar a la inclusión de los estudiantes con discapacidad, en entornos universitarios presenciales normalizados, por parte de sus compañeros, es independiente de las actitudes personales hacia la discapacidad. 
- H02: La intención de apoyar a la inclusión de los estudiantes con discapacidad, en entornos universitarios presenciales normalizados, por parte de sus compañeros, es independiente de la norma social dominante en relación con la discapacidad.

- H03: La intención de apoyar a la inclusión de los estudiantes con discapacidad, en entornos universitarios presenciales normalizados, por parte de sus compañeros, es independiente del control percibido.

- H04: La intención de apoyar a la inclusión de los estudiantes con discapacidad, en entornos universitarios presenciales normalizados, por parte de sus compañeros, no puede ser explicada en función de los postulados de la Teoría del Comportamiento Planeado (TPB).

- H05: No existen diferencias entre hombres y mujeres en los aspectos estudiados en este trabajo (actitudes, norma social y control percibidos, e intención de apoyo a la inclusión).

- H06: No existen diferencias entre las personas que conviven con personas con discapacidad en los aspectos estudiados en este trabajo (actitudes, norma social y control percibidos, e intención de apoyo a la inclusión).

\section{Método}

\section{Muestra}

Se ha realizado una encuesta a 164 jóvenes universitarios en el ámbito de las Ciencias Económicas y Empresariales de la Universidad de A Coruña, como prueba piloto para esta primera valoración de actitudes e intenciones de apoyo a la discapacidad por parte de los estudiantes de las titulaciones de Licenciado en Economía, Licenciado en Administración de Empresas y Diplomado en Estudios Empresariales. Previamente a la realización del trabajo de campo, se diseñó un cuestionario que fue testado, valorado y depurado por cinco profesores de la Facultad de Economía y Empresa de la Universidad de A Coruña, expertos en la aplicación de la técnica PLS (Partial Least Squares), cuyo objetivo es la predicción de las variables latentes, en el contexto de la Teoría del Comportamiento Planeado.

La aleatoriedad del proceso de selección aseguró la representatividad de la muestra y, por ello, la posibilidad de inferir los resultados al conjunto de la población objetivo. La media de edad fue de 22 años. El error aleatorio, a partir del supuesto de máxima indeterminación $(p=q=50)$ y con un margen de confianza de $95,5 \%$ fue de $5,3 \%$. El estudio fue realizado en mayo de 2010 . La estructura de la muestra puede verse en la Tabla 1, en la que se han recogido las variables de clasificación más representativas.

TABLA 1. Estructura de la Muestra

\begin{tabular}{|c|c|c|}
\hline Variable & Categoría & $\%$ \\
\hline \multirow[t]{3}{*}{ Genero } & Hombre & 32,32 \\
\hline & Mujer & 67,68 \\
\hline & Total & 100,0 \\
\hline \multirow[t]{4}{*}{$\begin{array}{l}\text { Estudios universitarios } \\
\text { (por titulación) }\end{array}$} & $\begin{array}{l}\text { Licenciado en Administración y } \\
\text { Dirección de Empresas (ADE) }\end{array}$ & 21,95 \\
\hline & Licenciado en Economía & 10,37 \\
\hline & $\begin{array}{l}\text { Diplomado en Estudios } \\
\text { Empresariales }\end{array}$ & 67,68 \\
\hline & Total & 100,0 \\
\hline \multirow[t]{5}{*}{ Edad } & $18-20$ & 21,95 \\
\hline & $21-23$ & 51,83 \\
\hline & $24-26$ & 21,34 \\
\hline & $>27$ & 4,88 \\
\hline & Total & 100,0 \\
\hline \multirow[t]{2}{*}{ Nivel educativo padre } & Ninguno & 0,61 \\
\hline & Primarios & 42,07 \\
\hline
\end{tabular}




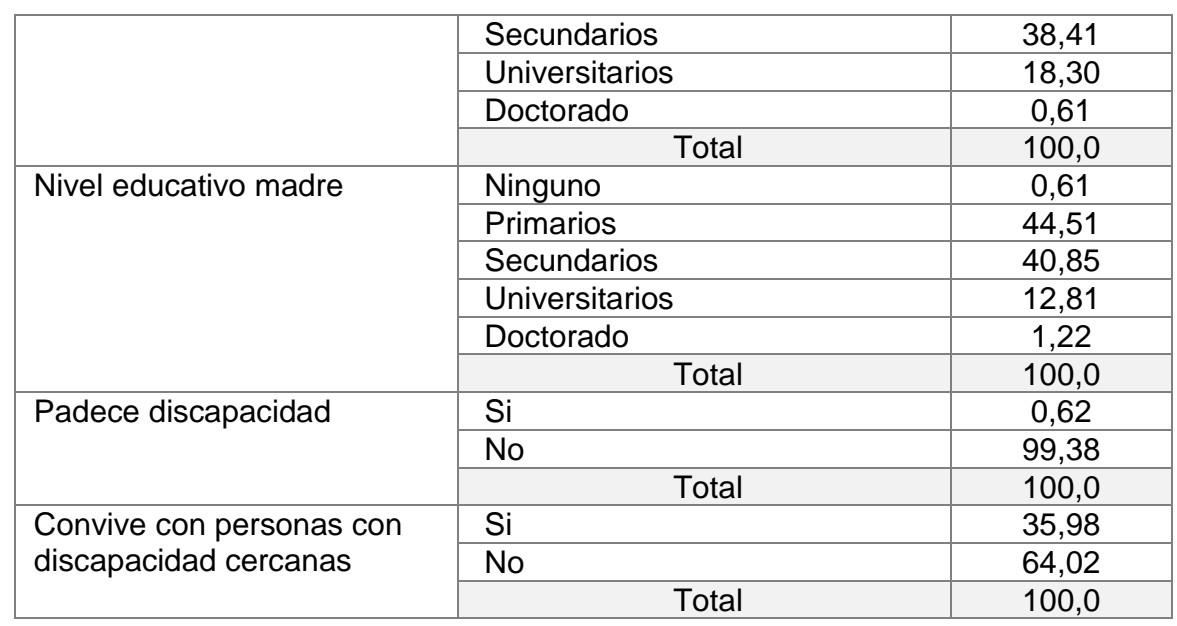

La polarización de la muestra hacia las mujeres responde a dos cuestiones: la mayor presencia como matriculadas en todas las titulaciones impartidas en el centro y el grado mayor de asistencia a clase de las chicas. La proporción de mujeres en las titulaciones estudiadas, durante el curso $2010-2011$ era del $60,82 \%$ en la licenciatura en administración y dirección de empresas, del $54,51 \%$ en la licenciatura en economía y del $56,55 \%$ en la diplomatura en estudios empresariales, como promedio la tasa de mujeres era del $58,09 \%$, según datos del Instituto Nacional de Estadística (INE). La mayor presencia de mujeres se refleja incrementada en la muestra debido a que la asistencia a clase de ellas es mayor, especialmente en períodos de exámenes, como ha ocurrido en esta ocasión.

\section{Instrumentos}

En cuanto a la selección de las variables observables, variables latentes y escalas de medida, hemos comenzado el cuestionario con una introducción formal explicando el tema de la investigación, las instituciones que la estaban llevando a cabo y los requisitos para cubrir el mismo. Los jóvenes universitarios han respondido con su grado de acuerdo/desacuerdo a ciertos ítems o indicadores que representan los factores determinantes de la intención a ayudar a personas con cierto grado de discapacidad. Las respuestas correspondían a una escala tipo Likert en la que se valoraba este grado de acuerdo o desacuerdo del 1 al 5 , representando el nivel 1 un desacuerdo absoluto y el nivel 5 un acuerdo total.

Según la literatura (Churchill, 1979), para la fiabilidad de la medición de las variables latentes se precisa de múltiples indicadores, ítems o variables observables, que en nuestro caso son recogidos en la tabla 2.

\section{Procedimiento}

Los datos se han recogido durante el mes de mayo de 2010 de forma presencial, entre los estudiantes que asistían a clase, mediante el siguiente sistema: en cada uno de los cursos de todas las titulaciones se ha pasado una vez, en clase, siendo el profesor el que repartía los cuestionarios entre los estudiantes, que los respondían y devolvían cumplimentados al responsable de la docencia en ese momento. El anonimato estuvo garantizado en todo momento, ya que los propios estudiantes encuestados introducían el cuestionario cubierto en un sobre, sin que el profesor pudiese en ningún momento identificar a quien pertenecía. Este procedimiento nos ha permitido acceder a todos los cursos de todas las titulaciones, si bien tiene el sesgo de que solamente ha sido cubierto por los estudiantes que voluntariamente han querido hacerlo, entre los que se encontraban en clase en ese momento. Es conveniente resaltar que en el momento de la 
recogida de datos el número de estudiantes presentes en las aulas no era tan numeroso (final de curso) como en otras épocas del curso. La fiabilidad del instrumento la hemos realizado calculando del Alfa de Cronbach. Para dar respuesta al objetivo que hemos formulado, se ha realizado esta investigación desde dos aspectos complementarios: por una parte se ha hecho un análisis de carácter descriptivo, para identificar cuáles son los factores determinantes que influyen en la intención de ayudar a los miembros con discapacidad. Por otra parte, se ha efectuado un estudio de comparación de medias, atendiendo al género y la convivencia o no con alguna persona con discapacidad. La metodología aplicada ha sido la de ecuaciones estructurales y análisis de comparación de medias.

\section{Resultados}

La fiabilidad de la escala de medida es la adecuada. La aplicación del Alfa de Cronbach arroja un valor de 0,874 para el conjunto de los ítems. Los valores desglosados según las variables latentes a que pertenecen se recogen en la tabla 2, así como los estadísticos descriptivos de cada uno de los ítems. Los valores del estadístico Alfa de Cronbach son aceptables para todos los constructos, excepto para Actitud, esto es debido a que se han medio aspectos de naturaleza muy diversa, de hecho al subdividir la actitud en dos grupos (ítems A4, A6 y A7 y A1, A2, A3, A5, A8 y A9) los valores aumentan a 0,650 y 0,642 que, sin ser muy elevado, mejoran sustancialmente.

TABLA 2. Indicadores relativos a la Actitud hacia la inclusión. Estadísticos descriptivos y fiabilidad de los constructos

\begin{tabular}{|c|c|c|c|c|}
\hline $\begin{array}{l}\text { Variable } \\
\text { Latente }\end{array}$ & Item & Media & D. Típica & $\begin{array}{c}\text { Alfa de } \\
\text { Cronbach }\end{array}$ \\
\hline \multirow{9}{*}{ 号 } & $\begin{array}{l}\text { A1 - Reconozco que no me he preocupado lo suficiente por } \\
\text { este tema }\end{array}$ & 3,232 & 1,100 & \multirow{9}{*}{0,3480} \\
\hline & $\begin{array}{l}\text { A2 - Honestamente, creo que es un problema con el que no } \\
\text { tengo nada que ver }\end{array}$ & 1,878 & 0,919 & \\
\hline & $\begin{array}{l}\text { A3 - Lo mejor que se puede hacer es crear centros especiales } \\
\text { para personas con discapacidad y atenderlos allí }\end{array}$ & 2,378 & 1,120 & \\
\hline & $\begin{array}{l}\text { A4 - Creo que es muy gratificante dedicar algo de tiempo propio } \\
\text { a la ayuda a los demás }\end{array}$ & 3,829 & 0,995 & \\
\hline & $\begin{array}{l}\text { A5 - El problema de las personas con discapacidad sólo afecta } \\
\text { a parte de la sociedad }\end{array}$ & 1,878 & 0,958 & \\
\hline & $\begin{array}{l}\text { A6 - Nos afecta a todos de alguna manera, aunque no la } \\
\text { padezcamos }\end{array}$ & 4,122 & 0,989 & \\
\hline & $\begin{array}{l}\text { A7 - En mi familia son muy sensibles hacia la inclusión de las } \\
\text { personas con discapacidad }\end{array}$ & 3,348 & 1,048 & \\
\hline & $\begin{array}{l}\text { A8 - En mi familia nunca ha habido personas con } \\
\text { discapacidad, por eso no me he preocupado de ello }\end{array}$ & 1,994 & 1,071 & \\
\hline & $\begin{array}{l}\text { A9 - Es un problema que debe afrontar e intentar resolver } \\
\text { quienes la padecen y/o su familia }\end{array}$ & 1,878 & 1,084 & \\
\hline \multirow{6}{*}{ 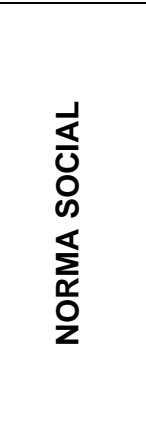 } & $\begin{array}{l}\text { N1 - Mis padres me han enseñado que cualquiera puede llegar } \\
\text { a ser una persona con discapacidad y necesitar de los demás }\end{array}$ & 4,091 & 0,996 & \multirow{6}{*}{0,8060} \\
\hline & $\begin{array}{l}\text { N2 - En la universidad los profesores se preocupan los las } \\
\text { personas con discapacidad }\end{array}$ & 2,921 & 0,966 & \\
\hline & $\begin{array}{l}\text { N3 - En la universidad los estudiantes se preocupan pos las } \\
\text { personas con discapacidad }\end{array}$ & 2,860 & 0,843 & \\
\hline & $\begin{array}{l}\text { N4 - En mi familia, desde pequeño me han enseñado que debo } \\
\text { ayudar a quienes me necesiten }\end{array}$ & 4,110 & 0,858 & \\
\hline & $\begin{array}{l}\text { N5 - En el colegio, desde pequeño me han enseñado que debo } \\
\text { ayudar a quienes me necesiten }\end{array}$ & 3,835 & 0,992 & \\
\hline & $\begin{array}{l}\text { N6 - En el instituto me han enseñado que debo ayudar a } \\
\text { quienes me necesiten }\end{array}$ & 3,610 & 1,083 & \\
\hline
\end{tabular}




\begin{tabular}{|c|c|c|c|c|}
\hline & $\begin{array}{l}\text { N7 - Creo que la obligación de cualquier persona es ayudar a } \\
\text { otra que la necesite }\end{array}$ & 3,829 & 1,217 & \\
\hline \multirow{4}{*}{ 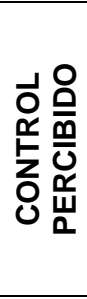 } & $\begin{array}{l}\text { C1 - Me siento capacitada/o para apoyar y ayudar a cualquier } \\
\text { persona con discapacidad }\end{array}$ & 3,488 & 1,054 & \multirow{4}{*}{0,8480} \\
\hline & $\begin{array}{l}\text { C2 - Creo que soy capaz de percibir las necesidades } \\
\text { especiales de las personas con discapacidad }\end{array}$ & 3,299 & 1,022 & \\
\hline & $\begin{array}{l}\text { C3 - Creo que sería capaz de ayudar a alguna persona con } \\
\text { discapacidad física }\end{array}$ & 3,537 & 0,903 & \\
\hline & $\begin{array}{l}\text { C4 - Creo que sería capaz de ayudar a alguna persona con } \\
\text { discapacidad psíquica o sensorial }\end{array}$ & 3,079 & 1,130 & \\
\hline \multirow{6}{*}{ 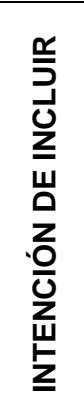 } & $\begin{array}{l}\text { I1 - Mi deseo es participar en organizaciones para apoyo a las } \\
\text { personas en riesgo de exclusión }\end{array}$ & 2,732 & 1,022 & \multirow{6}{*}{0,8170} \\
\hline & $\begin{array}{l}\text { I2 - Me gustaría participar en programas de ayuda a la inclusión } \\
\text { social }\end{array}$ & 3,024 & 1,051 & \\
\hline & $\begin{array}{l}\text { I3 - Quiero que las instituciones educativas promuevan } \\
\text { programas para la inclusión de personas con discapacidad }\end{array}$ & 3,665 & 1,041 & \\
\hline & $\begin{array}{l}14 \text { - Tengo intención de ayudar a la inclusión de las personas } \\
\text { con discapacidad }\end{array}$ & 3,018 & 1,054 & \\
\hline & $\begin{array}{l}\text { I5 - Si la universidad propusiese programas de apoyo a los/as } \\
\text { discapacitados/as, yo me apuntaría }\end{array}$ & 3,244 & 1,016 & \\
\hline & I6 Intento ayudar a alguna persona con discapacidad & 2,909 & 1,101 & \\
\hline
\end{tabular}

Para evaluar las diferencias en medias, según los grupos analizados, se ha utilizado la prueba $\mathrm{t}$ de Student, con un probabilidad ${ }^{* *} \mathrm{p}<0.05$, para rechazar la hipótesis nula (igualdad de medias). Previamente se ha realizado la prueba de Levene, asumiendo varianzas iguales en los ítems estudiados.

\section{Análisis de los constructos}

Hemos construido tres modelos de ecuaciones estructurales (uno para cada constructo), relacionando cada uno de ellos con la intención de apoyo a la inclusión. En la figura 1 se recoge el constructo actitud (los demás se omiten por ser similares) y el resumen los resultados para los cuatro constructos se recoge en la tabla 3, en la cual se muestran los pesos de regresión para explicar la variable latente, los niveles de ajuste mediante los indicadores Chi-cuadrado y CFI (Comparative Fit Index, índice comparativo de ajuste). Todos los valores son aceptables y todos los ítems se muestran altamente significativas, indicando la correcta construcción de las variables latentes. Sin embargo, conviene llamar la atención sobre los signos negativos de algunos coeficientes en el constructo actitud, que indican la distinta naturaleza de los ítems que lo componen, tal como ya se había señalado. 
FIGURA 1. Relación entre la variable latente actitud y los ítems que la forman

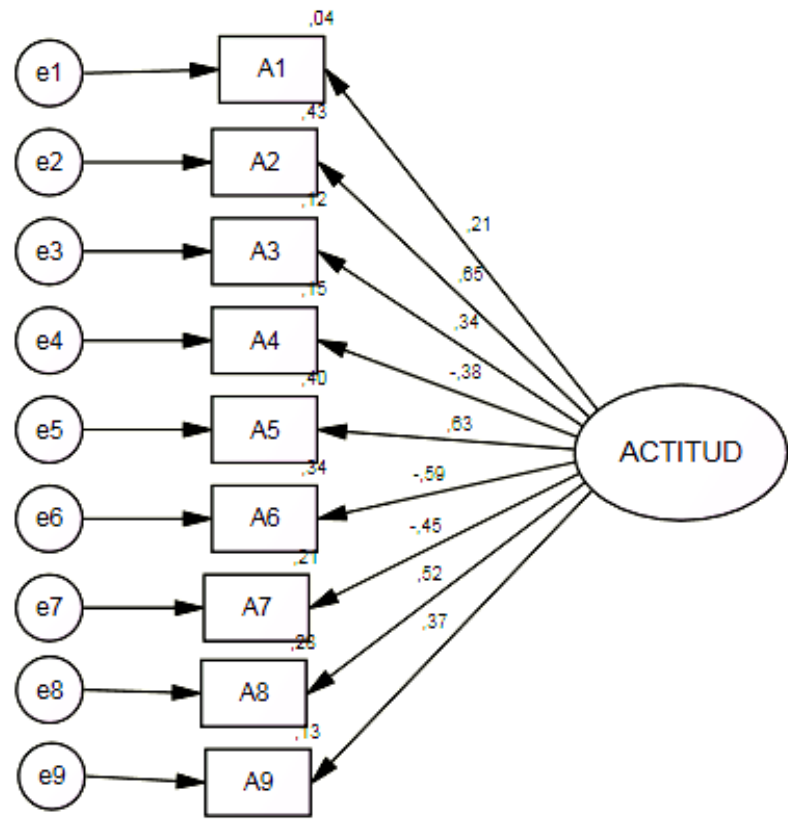

TABLA 3. Relación entre los ítems y los constructos

\begin{tabular}{|c|c|c|c|}
\hline Ítem & $\begin{array}{c}\text { Estimadores } \\
\text { Estandarizados* }\end{array}$ & $\begin{array}{c}\text { Ajuste del } \\
\text { Modelo (CFI) }\end{array}$ & $\begin{array}{l}\text { Chi-Cuadrado (Grados } \\
\text { Libertad) (probabilidad) }\end{array}$ \\
\hline $\mathrm{A} 1^{*}$ & 0,210 & \multirow{9}{*}{0,748} & \multirow{9}{*}{$126,091(\mathrm{gl}=28)(\mathrm{p}=0.000)$} \\
\hline $\mathrm{A} 2^{* *}$ & 0,653 & & \\
\hline $\mathrm{A}^{*}$ & 0,344 & & \\
\hline $\mathrm{A} 4^{* *}$ & $-0,383$ & & \\
\hline$A 5^{* *}$ & 0,635 & & \\
\hline $\mathrm{A} 6^{* \star}$ & $-0,587$ & & \\
\hline$A 7^{*}$ & $-0,453$ & & \\
\hline$A 8^{* *}$ & 0,525 & & \\
\hline $\mathrm{A} 9^{* *}$ & 0,367 & & \\
\hline $\mathrm{N} 1^{\star * *}$ & 0,597 & \multirow{7}{*}{0,74} & \multirow{7}{*}{$129,545(\mathrm{gl}=14)(\mathrm{p}=0.000)$} \\
\hline $\mathrm{N}^{* * *}$ & 0,404 & & \\
\hline $\mathrm{N}^{* * *}$ & 0,380 & & \\
\hline$N 4^{* * *}$ & 0,746 & & \\
\hline N5 ${ }^{* * *}$ & 0,860 & & \\
\hline$N 6^{\star \star \star}$ & 0,776 & & \\
\hline$N 7^{* * *}$ & 0,477 & & \\
\hline $\mathrm{C} 1^{* * *}$ & 0,743 & \multirow{4}{*}{0,991} & \multirow{4}{*}{$4,552(g \mid=2)(p=0,103)$} \\
\hline $\mathrm{C}^{\star \star \star}$ & 0,807 & & \\
\hline $\mathrm{C}^{* * *}$ & 0,857 & & \\
\hline $\mathrm{C} 4^{* * *}$ & 0,682 & & \\
\hline $11^{\star \star \star}$ & 0,734 & \multirow{6}{*}{0,897} & \multirow{6}{*}{$42,718(g l=9)(p=0,000)$} \\
\hline $12^{\star \star *}$ & 0,695 & & \\
\hline $13^{\star \star *}$ & 0,555 & & \\
\hline $14^{\star \star \star}$ & 0,805 & & \\
\hline $15^{\star \star *}$ & 0,651 & & \\
\hline $16^{\star \star \star}$ & 0,517 & & \\
\hline
\end{tabular}


A la vista de los datos de la tabla 2, se comprueba que los estudiantes universitarios tienen actitudes favorables en todos los constructos analizados. Sin embargo, para dar respuesta a nuestras preguntas y testar las hipótesis planteadas, hemos relacionado los constructos actitudes, norma social y control percibido con la intención de apoyo a la inclusión. Para ello también hemos utilizado modelos de ecuaciones estructurales. La figura 2 sirve como ejemplo de lo que hemos planteado.

FIGURA 2. Control percibido como variable explicativa de la intención de apoyo a la inclusión

$$
\begin{gathered}
\text { Novo-Corti \& Muñoz-Cantero } \\
\text { Chi squared:95,108 } \\
\text { Dgrees of fredom: } 34 \\
\text { CF: } 910
\end{gathered}
$$

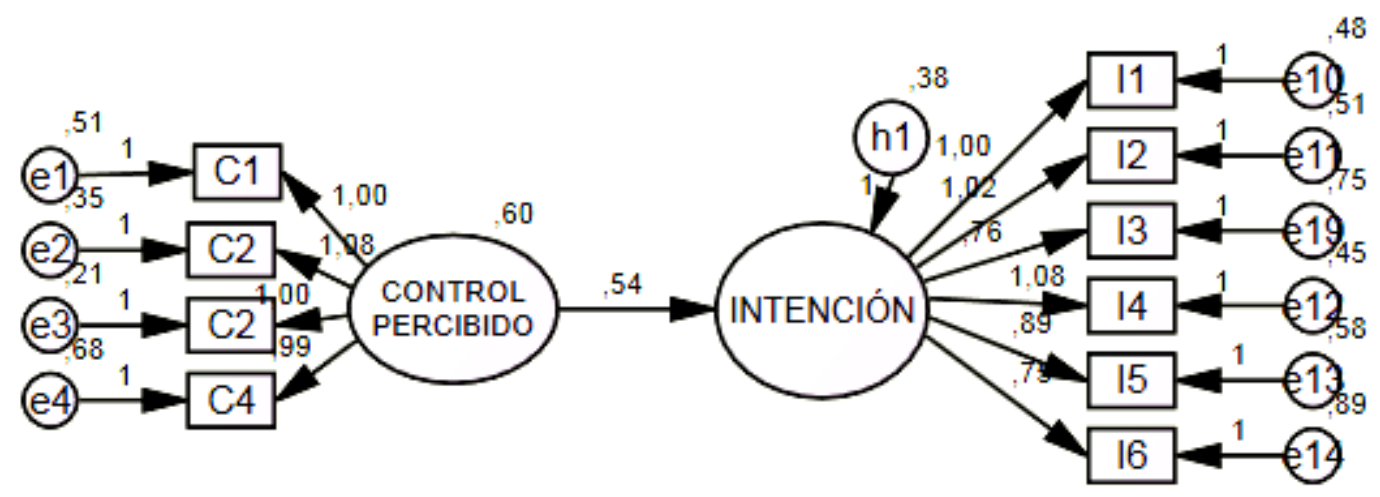

En todos los casos analizados se rechazan las hipótesis nulas (H01, H02, H03 y H04), por tanto, las respuestas a nuestras preguntas son necesariamente afirmativas. La actitud, la norma social y el control percibido explican la intención de apoyo a la inclusión, al ser todos los constructos significativos y el ajuste del modelo dentro de los parámetros aceptables (CFI (Actitudes)=0,8; CFI (Norma social)=0,78; CFI (Control percibido)=0,78) y, por eso podemos considerar que la Teoría del Comportamiento Planeado es adecuada para valorar la misma.

\section{Análisis de comparación de medias}

La importancia del género en lo relativo a actitudes hacia la discapacidad se encuentra en numerosos trabajos, entre los que cabe citar los de Weisel y Florian (1990), Thistlethwaite y Ewart (2003), Moreno et al (2006), Goreczny et al (2011) y Scior (2011). En nuestro caso, hemos

\begin{tabular}{|c|c|c|c|c|c|c|}
\hline \multirow{3}{*}{$\begin{array}{l}\text { Contraste t Student para comparación } \\
\text { medias* }\end{array}$} & \multicolumn{6}{|c|}{ Prueba t para la igualdad de medias } \\
\hline & \multirow{2}{*}{$t(g \mid=161)$} & \multirow{2}{*}{$\begin{array}{c}\text { Sig. } \\
\text { (bilateral) }\end{array}$} & \multirow{2}{*}{$\begin{array}{l}\text { Diferencia } \\
\text { de medias }\end{array}$} & \multirow{2}{*}{$\begin{array}{l}\text { Error típ. de } \\
\text { la diferencia }\end{array}$} & \multicolumn{2}{|c|}{$\begin{array}{l}95 \% \text { Intervalo de confianza } \\
\text { para la diferencia }\end{array}$} \\
\hline & & & & & Inferior & Superior \\
\hline $\begin{array}{l}\text { A2 - Honestamente, creo que es un problema } \\
\text { con el que no tengo nada que ver }\end{array}$ & 1,978 & ,050 & ,302 & ,153 & 000 & ,604 \\
\hline
\end{tabular}
encontrado diferencias estadísticamente significativas en los siguientes ítems recogidos en la tabla 4.

TABLA 4. Comparación de medias por género 


\begin{tabular}{|l|c|c|c|c|c|c|}
\hline $\begin{array}{l}\text { A8 - En mi familia nunca ha habido personas } \\
\text { con discapacidad, por eso no me he } \\
\text { preocupado de ello }\end{array}$ & 1,986 &, 049 &, 347 &, 175 &, 002 &, 691 \\
\hline $\begin{array}{l}\text { I3 - Quiero que las instituciones educativas } \\
\text { promuevan programas para la inclusión de } \\
\text { personas con discapacidad }\end{array}$ & $-3,008$ &, 003 &,- 511 &, 170 &,- 846 &,- 175 \\
\hline
\end{tabular}

*Prueba de comparación de medios para muestras independientes.

Las diferencias se encuentran en los constructos actitud e intención, en consonancia con lo indicado por Weisel y Florian (1990). El primer ítem (A2) (con medias de 1,77 para mujeres y 2,08 para hombres) indica que las mujeres están más sensibilizadas hacia la discapacidad. Por otra parte, habida cuenta de que hombres y mujeres comparten las mismas familias, el ítem A8 refleja la percepción diferente en hombres y mujeres sobre la posición de sus familias, consideradas más implicadas por parte de las mujeres (las medias de hombres y mujeres son 2,21 y 1,86 respectivamente).

El ítem recogido 13 es muy relevante, en nuestra opinión, ya que los encuestados expresan claramente sus deseos de apoyar las políticas inclusivas o no. Las diferencias por género, indican nuevamente el mayor deseo de las mujeres de favorecer el apoyo a las personas con discapacidad, pues son ellas precisamente las que manifiestan mayor acuerdo con la afirmación propuesta (3,84 frente a 3,33 de los hombres).

La convivencia con personas con discapacidad ha sido considerada una pieza importante para generar actitudes proinclusivas, tal como ha sido puesto de manifiesto por diversos trabajos, entre los que destacan los de Birkel (1987), Philp et al (1987), Tabatabainia (2003) y Nuñez y Rodríguez (2005). El estudio comparativo entre grupos de personas que pertenezcan o no a dicho colectivo, arroja resultados con diferencias significativas en medias para los ítems recogidos en la tabla 4.

Todo esto nos permite rechazar la hipótesis $\mathrm{H} 05$, ya que se ha comprobado que existen diferencias estadísticamente significativas en medias entre géneros.

TABLA 5. Comparación de medias para convivencia con personas con discapacidad

\begin{tabular}{|c|c|c|c|c|c|c|c|}
\hline \multirow{3}{*}{ ÍTEM } & \multicolumn{7}{|c|}{ Prueba $T$ para la igualdad de medias } \\
\hline & \multirow[t]{2}{*}{$\mathrm{t}$} & \multirow[t]{2}{*}{ gl } & \multirow[t]{2}{*}{$\begin{array}{c}\text { Sig. } \\
\text { (bilateral) }\end{array}$} & \multirow[t]{2}{*}{$\begin{array}{l}\text { Diferencia } \\
\text { de medias }\end{array}$} & \multirow[t]{2}{*}{$\begin{array}{l}\text { Error típ. de } \\
\text { la diferencia }\end{array}$} & \multicolumn{2}{|c|}{$\begin{array}{l}95 \% \text { Intervalo de } \\
\text { confianza para la } \\
\text { diferencia }\end{array}$} \\
\hline & & & & & & Inferior & Superior \\
\hline $\begin{array}{l}\text { A2 - Honestamente, creo que es un } \\
\text { problema con el que no tengo nada } \\
\text { que ver }\end{array}$ & $-3,439$ & 162 & ,001 &,- 498 & ,145 &,- 784 &,- 212 \\
\hline $\begin{array}{l}\text { A5 - El problema de personas con } \\
\text { discapacidad sólo afecta a parte de la } \\
\text { sociedad }\end{array}$ & $-2,024$ & 162 & ,045 &,- 313 & ,154 &,- 617 &,- 008 \\
\hline $\begin{array}{l}\text { A7 - En mi familia son muy sensibles } \\
\text { hacia la inclusión de las personas con } \\
\text { discapacidad }\end{array}$ & 3,274 & 162 & 001 & ,543 & 166 & ,215 & ,870 \\
\hline $\begin{array}{l}\text { A8 - En mi familia nunca ha habido las } \\
\text { personas con discapacidad, por eso } \\
\text { no me he preocupado de ello }\end{array}$ & $-6,166$ & 162 & ,000 &,- 970 & ,157 & $-1,281$ &,- 659 \\
\hline
\end{tabular}




\begin{tabular}{|l|c|c|c|c|c|c|c|}
\hline $\begin{array}{l}\text { C4 - Creo que sería capaz de ayudar a } \\
\text { alguna persona con discapacidad } \\
\text { psíquica o sensorial }\end{array}$ & $-1,988$ & 162 &, 049 &,- 362 &, 182 &,- 722 &,- 002 \\
\hline
\end{tabular}

Los ítems que muestran diferencias significativas en medias, excepto uno, relativo al control percibido, son todos ellos determinantes del constructo actitud aunque se pueden agrupar en tres categorías: las relativas a la actitud personal (ítems 1 y 2 , de la tabla 4), las relativos a la interacción con la familia (ítems 3 y 4, de la tabla 4) y el último ítem relacionado con la propia capacidad.

La primera categoría indica que quienes conviven con personas con discapacidad se sienten más implicados en esta cuestión (las medias son de 1,56 - 1,68 para los que conviven y 2,06 1,99 , para los que no tienen contacto directo con personas con discapacidad, en relación con los ítems 1 y 2).

El posicionamiento familiar también es más favorable en el caso de que se conviva con personas con discapacidad, como era de esperar (las medias son de 3,69-1,37 y 3,15-2,34, según haya personas con discapacidad en el entorno familiar o no, respectivamente, para los ítems 3 y 4). Conviene matizar que el sentimiento familiar es más fuerte que el individual, de ahí las mayores diferencias en medias.

En cuanto a la capacidad de ayuda a las personas con discapacidad, no se han detectado diferencias estadísticamente significativas en medias cuando son preguntados por la discapacidad física, por lo que podemos intuir cierta homogeneidad en la autopercepción de la capacidad para apoyar a este colectivo. Sin embargo, la situación es completamente diferente, cuando se les pregunta si se sienten capacitados para ayudar a una persona con discapacidad psíquica. Es precisamente en este caso, donde se pone de manifiesto la mayor capacidad o competencia para apoyar a las personas con discapacidad psíquica (las medias son de 2,85 y 3,21). Curiosamente son precisamente las personas que conviven con el problema de la discapacidad, las que manifiestan menor competencia. En nuestra opinión, esto es debido a un mayor conocimiento de la complejidad del problema, lo que indica la necesidad de ayuda por parte de instituciones que pongan a su disposición profesionales competentes.

Todo esto nos permite rechazar las hipótesis $\mathrm{H} 05$ y $\mathrm{H} 06$ ya que se ha comprobado que existen diferencias estadísticamente significativas en medias en función del género y convivencia con personas con discapacidad. Como consecuencia las respuestas a las preguntas 5 y 6 son, necesariamente afirmativas.

\section{Conclusiones}

La actitud del estudiantado universitario es favorable a la inclusión (Surriá Martínez, 2011), la norma social está muy fuertemente arraigada y el control percibido es bastante elevado. La intención de apoyo a los/as estudiantes con discapacidad es claramente positiva; sin embargo, sólo un 5,5\% de los/as encuestados/as han manifestado su determinación a ayudar, y un 27,4\% están "bastante dispuestos/as" a hacerlo. Se ha comprobado que la Teoría del Comportamiento Planeado ha focalizado correctamente los puntos clave para explicar la intención de apoyo a la inclusión, por tanto, permite focalizar los campos de actuación de las políticas inclusivas sobre las tres variables latentes consideradas. La respuesta afirmativa a las seis preguntas que nos hemos planteado nos lleva proponer acciones que mejoren los tres aspectos: actitudes, norma social y control percibido, tomando en consideración las características propias del colectivo al que van 
dirigidas, especialmente, el género o la proximidad convivencial con la discapacidad, ya que, tal como se ha comprobado, son variables importantes para determinar la intención de apoyo a la inclusión. En cuanto a la primera, se ha puesto de manifiesto que las mujeres muestran mayor sensibilidad tanto hacia la discapacidad, como hacia la necesidad de promover políticas educativas inclusivas en el entorno universitario (aunque esta necesidad es también compartida, en menor medida, con los hombres).

Los resultados visibilizan la importancia de la familia en la generación de valores inclusivos y en sensibilidad hacia la discapacidad, especialmente manifestada en el caso de presencia de algún miembro afectado. Considerando, además, que la convivencia se ha revelado como una variable diferenciadora en intenciones, la familia se sitúa como un objetivo importante de actuación ${ }^{3}$.

Las conclusiones alcanzadas en este trabajo, plasmadas en las respuestas afirmativas a las seis preguntas formuladas, apuntan hacia el probable éxito de las políticas inclusivas, ya que los/as estudiantes/as se han mostrado solícitos/as para la participación en organizaciones o programas de actividades promovidas por los centros universitarios o instituciones de rango superior. Por otra parte los previsibles resultados favorables tendrían implicaciones en ambos sentidos: tanto para los/as estudiantes con discapacidad como para sus compañeros/as, los/as primeros/as por poder realizar una vida lo más normalizada posible y los/as segundos/as por sentirse gratificados/as (tal como ellos mismos manifiestan) con su colaboración. Se detecta una mayor dificultad de capacidad de los estudiantes para apoyar a sus compañeros con discapacidad psíquica, especialmente acusada en el caso de aquellos que conviven directamente con el problema.

Nuestras principales recomendaciones, se dirigen hacia el diseño e implementación de políticas educativas inclusivas de corte transversal y aplicadas en el ámbito familiar y desde las primeras etapas de estudio (los estudiantes han manifestado la importancia la familia, del colegio y el instituto -ítems N4, N5 y N6-). Sin embargo, subrayamos su importancia en el ámbito universitario, habida cuenta de que los estudiantes han manifestado su intención en participar en dichas acciones (15). Finalmente, sugerimos que se ponga especial énfasis en la formación y apoyo para los estudiantes con discapacidad psíquica.

\section{Limitaciones del estudio y futuras líneas de investigación}

Las principales limitaciones de este trabajo proceden de dos fuentes: por una parte, se ha considerado muy conveniente completar el mismo con un análisis de tipo cualitativo que permita matizar y reforzar las conclusiones. Por otra parte, las relacionadas con la muestra. Algunas de ellas ya se han señalado, otras están relacionadas con el procedimiento de recogida de datos (presencial en aulas), que ha sesgado la composición de la muestra al obviar a los estudiantes que no suelen acudir al centro. Sin embargo, en este apartado, quisiéramos incidir en la necesidad ampliar este estudio a otros centros y universidades, al objeto de que los resultados puedan ser generalizables con mayor confianza. La elección del centro no ha sido arbitraria y está relacionada con las futuras líneas de investigación. Hemos considerado que la actitud inclusiva de los estudiantes de economía y empresa, así como los de ciencias empresariales es muy importante

\footnotetext{
${ }^{3}$ Finalmente, un dato a tener en cuenta es el resultado obtenido al examinar las actitudes ante las personas con discapacidad en función de si conocen o mantienen contacto con alguna persona discapacitada o no, de forma que, observamos que los estudiantes encuestados que tienen la experiencia de convivencia directa con personas con alguna discapacidad mantienen una actitud ante ellas más positiva que los que no la tienen" (Surriá Martínez, 2011: 212-213).
} 
para la inclusión socio-laboral de las personas con discapacidad. Por tal motivo, y dado que esta es nuestra futura línea de investigación, hemos considerado muy importante tener una valoración de las actitudes de aquellos que, previsiblemente, en el futuro no sólo tendrán responsabilidades en la gestión y dirección de las empresas, sino también en el diseño e implementación de políticas económicas y laborales.

\section{Referencias bibliográficas}

AJZEN, I. (1991). The theory of planned behavior. Organizational Behavior and Human Decision Processes, 50, 179-211.

Alonso, M.J., NAVARRo, R y Ridón, V. (2007). Actitudes hacia la diversidad en estudiantes universitarios. Jornadas de Fomento de la investigación. Extraído el 4 de agosto de 2010, desde http://www.uji.es/bin/publ/edicions/jfi13/44.pdf

ALTMAN, B.M. (1986). Definitions of disability in empirical research: Is the use of an administrative definition co-opting the results of disability research?. Paper presented at the Annual Meetings of American Sociological Association, Washington, DC.

AlPONTE-HERNANDEZ, E. (2008). Desigualdad, inclusión y equidad en la educación superior en América Latina y el Caribe: tendencias y escenario alternativo en el horizonte 2021. En Lucía Gazzola, A. and Didriksson Takayanaqui, A. (Eds.) Tendencias de la educación superior en América Latina y el Caribe. UNESCO, Instituto Internacional para la Educación Superior en América Latina y el Caribe.

ASPINALL, P. J. (1997). The conceptual basis of ethnic group terminology and classifications. Social Science \& Medicine, 45, 5, 689-698.

BARNES, C., \& MERCER, G. (Eds.). (2004). Implementing the social model of disability: Theory and research. Leeds: Disability Press.

BARTON, L. (2009). Estudios sobre discapacidad y la búsqueda de la inclusividad. Observaciones. Revista de Educación, 349, 137-152.

BICKENBACH, J.E., Chatterji, S., Badley, E.M., and Üstün, T.B. (1999). Models of disablement, universalism and the international classification of impairments, disabilities and handicaps. Social Science \& Medicine, 48, 1173-1187.

BIRKEL, R.C. (1987). Toward a social ecology of the home-care household. Psychology and Aging, 2 (3), 294-301.

CANNAN, C. \& WARREN, C. (1997). Social action with children and families: A Community development approach to child and family welfare. Roudlege.

ChIROLEU, A. (2009). La inclusión en la educación superior como política pública: tres experiencias en América Latina. Revista Iberoamericana de Educación n.ํ 48/5-25 Extraído el 7 de septiembre de 2011 desde http://www.rieoei.org/deloslectores/2740Chiroleu.pdf

Churchill, G.A. (1979). A paradigm for developing better measures of marketing constructs. Journal of Marketing Research, 1, 1, 64-73.

CLORE, G.L. AND JEFFERY, K.M. (2007). Emotional role playing, attitude change, and attraction toward a disabled person. Journal of Personality and Social Psychology, 23, 1, 105-111.

Collins, A.J., GILBERTSON, D.T., SNYDER, J.J., ChEN, S. AND FOlEY, R.N. (2010). Chronic kidney disease awareness, screening and prevention: Rationale for the design of a public education program. Nephrology, 15 (SUPPL. 2), 37-42. 
COMUNICACIÓN DE LA COMISIÓN (2010). EUROPA 2020 Una estrategia para un crecimiento inteligente, sostenible e integrador 2020. Bruselas

DueñAS BueY, MaL. (2010). Educación Inclusiva. Revista Española de Orientación y Psicopedaogía. 21, 2, 358-366.

ERNST, C. \& Rogers, M.R. (2009). Development of the Inclusion Attitude Scale for High School Teachers. Detail. Journal of Applied School Psychology, 25, 3, 305-322.

FERNÁNDEZ BATANERO, J.Ma (2004). Necesidades Educativas Especiales en el contexto universitario español. Revista de la Educación Superior, Vol. XXXIII (3), 131.

FISHBEIN, M., \& AJZEN, I. (1974). Attitudes toward objects as predictors of single and multiple behavioral criteria. Psychological Review, 81, 59-74.

FUJIURA, G.T., \& RUTKOWSKI-KMITTA, V. (2001). Counting disability. In G.L. Albrecht, K.D. Seelman, \& M. Bury (Eds.), Handbook of disability studies. Thousand Oaks, CA: Sage. (pp. 69-96).

Goreczny, A.J., Bender, E.E., CARuso, G., And Feinstein, C.S. (2011). Attitudes toward individuals with disabilities: Results of a recent survey and implications of those results. Research in Developmental Disabilities, 32 (5), 1596-1609.

GRÖNVIK, L. (2009). Defining disability: effects of disability concepts on research outcomes. International. Journal of Social Research Methodology, 12, 1, 1-18.

Gross, B.H., \& HAHN, H. (2004). Developing issues in the classification of mental and physical disabilities. Journal of Disability Policy Studies, 15(3), 130-134.

HABER, L.D. (1967). Identifying the disabled: Concepts and methods in the measurement of disability. Social Security Bulletin, 30, 17-34.

HorRocks, J.L., White, G., AND RoBerTS, L. (2008). Principals' attitudes regarding inclusion of children with autism in pennsylvania public schools. Journal of Autism and Developmental Disorders, 38(8), 1462-1473.

Hahn, H., \& Pool Hegamin, A. (2001). Assessing scientific measures of disability. Journal of Disability Policy Studies, 12(2), 114-121.

INFANTE, M. Y GÓMEZ, V. (2004). Actitudes de los estudiantes de educación hacia la integración de personas con discapacidad y hacia la educación multicultural. Cultura y educación, 16, 4, 371384.

LANNEN, T.; BRoWN, D.; POWELL, H. (2002). Control of virtual environments for young people with learning difficulties.. Disability and Rehabilitation, 24, 11/12, 578-586.

LÁzaro Torrente, L.M., Tiana Ferrer, A. (Ed.). (2001) Problemas y desafíos para la educación en el s. XXI en Europa y América Latina. España: Universidad de Valencia.

LENOIR, R. (1974). Les Exclus: Un Francais sur Dix. Paris: Editions du Seuil.

LÓPEZ TORRIJO, M. (2009). La inclusion educativa de alumnos con discapacidades graves y permanentes en la Unión Europea. RELIEVE, 15, 1.

LUQUE, D.J. (2008). Valores y necesidades educativas especiales: Elementos para un estudio psicoeducativo. INFAD. International Journal of Developmental and Educational Psychology, 1, 125-136.

LUQUE, D.J. Y RodRIGUEZ, G. (2008). Alumnado Universitario con Discapacidad: Elementos para la reflexión psicopedagógica. Revista Española de Orientación y Psicopedagogía, 19, (3), 270281.

LUQUe PARRA, J. Y LUQUE-RoJAS, Ma.J. (2010). Acción tutorial t discapacidad: sugerencias de trabajo en el aula. Revista Española de Orientación y Psicopedagogía, 21, 3, 633 - 642 
Martin, J., MeltZer, H., AND ElLiOT, D. (1988). The prevalence of disability y Psicopedagogía among adults. London: Office of Population Censuses and Surveys.

Moreno, J.F, Rodríguez, I.R., SAldañA, D. y AGUILERA, A. (2006) Actitudes ante la discapacidad en el alumnado universitario matriculado en materias afines. Revista Iberoamericana de Educación, 40/5. Recuperado el 12 de Julio de 2012, de http://www.rieoei.org/investigacion/1491Moreno.pdf

MoRIÑA, A. (2008). ¿Cómo hacer que un centro educativo sea inclusive? Análisis del diseño, desarrollo y resultados de un program formative. Revista de Investigación Educativa, 23, 1, 165-189.

McCaul, K.D.; Sandgren, A.K.; O'NeILL, H.K.; Hinsz, V.B. (1993) The Value of the Theory of Planned Behavior, Perceived Control, and Self-Efficacy Expectations for Predicting HealthProtective Behaviors. Basic and Applied Social Psychology, 14, 2, 231-252.

MuelleR, S.C.; JACKSON, G.M.; Dhalla, R.; DATSOPOUlos, S.; Hollis, C.P. (2006). Enhanced Cognitive Control in Young People with Tourette's Syndrome. Detail Current Biology, 16, 6, 570-573.

MurRay, C., LOMBARDI, A., \& WrEN, C.T. (2011). The effects of disability-focused training on the attitudes and perceptions of university staff. Remedial and Special Education, 32(4), 290-300.

NAGI, S.Z. (1979). The concept and measurement of disability. In E.D. Berkowitz (Ed.), Disability policies and government programs. New York: Praeger. (pp. 1-15).

NemmE, H.E.; WHITE, K.M. (2010). Texting while driving: Psychosocial influences on young people's texting intentions and behaviour. Accident Analysis and Prevention, 42, 4, 1257-1265.

NUÑEZ, B. Y RODRíGUEZ, L. (2004). Los hermanos de personas con discapacidad: una asignatura pendiente. Cochabamba 3243, Buenos Aires (1252) - Argentina ( $2^{\mathrm{a}}$ ed. 2005). Recuperado el 12 de Julio de 2012 de http://autismodiario.org/wp-content/uploads/2011/02/Los-hermanosde-personas-con-discapacidad.pdf

Oliver, M. (1996). Understanding disability. From theory to practice. New York: St. Martin's Press.

PALACIOS, A. (2008). El modelo social de discapacidad: orígenes, caracterización y plasmación en la Convención Internacional sobre los Derechos de las Personas con Discapacidad. Madrid: Ed. Cinca.

PAUL, S.N.; Frank, A.O.; HANSPAL, R.S; Groves, R. (2006). Exploring environmental control unit use in the age group 10--20 years. International Journal of Therapy \& Rehabilitation, 13, 11, 511-516.

Pelling, E.L. \& White, K.M. (2009). The Theory of Planned Behavior Applied to Young People's Use of Social Networking Web Sites. CyberPsychology and Behavior, 12, 6, 755-759.

PhilP, I., MCKeE, K.J., ARMStRong, G.K., BAllingeR, B.R., GiLhOOLY, M.L.M., Gordon, D.S., ET AL. (1997). Institutionalization risk amongst people with dementia supported by family careers in a scottish city. Aging and Mental Health, 1(4), 339-345.

Prescott, H.M. (2011). Student bodies, past and present. Journal of American College Health, 59(6), 464-469.

Rodriguez-Ascaso, A., Boticario, J.G., Finat, C., Del Campo, E., Saneiro, M., Alcocer, E., et AL. (2011). Inclusive scenarios to evaluate an open and standards-based framework that supports accessibility and personalisation at higher education. UAHCl'11 Proceedings of the $6^{\text {th }}$ International conference on Universal access in human-computer interaction: applications and services. Vol. Part IV, pp.612-621. Berlin, Heidelberg: Springer-Verlag. 
Sánchez Bravo, A.; Díaz Flores, C.; Sanhueza Henríquez, S.; Friz Carrillo, M. (2008) Percepciones y actitudes de los estudiantes de Pedagogía hacia la inclusión educativa. Estudios Pedagógicos XXXIV, 2, 169-178.

ScIOR, K. (2011). Public awareness, attitudes and beliefs regarding intellectual disability: A systematic review. Research in Developmental Disabilities, 32(6), 2164-2182.

SHAKESPEARE, T. (2005). Review article: Disability studies today and tomorrow. Sociology of Health and IIIness, 27(1), 138-148.

Sharma, U.; MoORE, D.; SONAWANE, S. (2009). Attitudes and concerns of pre-service teachers regarding inclusion of students with disabilities into regular schools in Pune, India. AsiaPacific. Journal of Teacher Education, 37, 3, 319-331.

SURRIÁ MARTínEZ, R. (2011) Análisis comparativo de las actitudes de los estudiantes hacia sus compañeros con discapacidad. Electronic Journal of Research in Educational Psychology, $9(1), 23,197-216$

SZEBEHELY, M., FRITZELL, J., AND LUNDBERG, O. (2001). Funktionshinder och välfärd. SOU 2001: 56 [Disability and welfare]. Stockholm: Fritzes.

Oliver, M. (1990). The politics of disablement. Basingstoke: Macmillan.

TABATABAINIA, M.M. (2003). Listening to families' views regarding institutionalization \& deinstitutionalization. Journal of Intellectual and Developmental Disability, 28(3), 241-259.

ThISTLETHWAITE, J.E. \& EWART, B.R. (2003). Valuing diversity: Helping medical students explore their attitudes and beliefs. Medical Teacher, 25(3), 277-281.

UNITED NATIONS (1990). Disability statistics compendium. New York: United Nations.

UNITED NATIONS (1996). Manual for development of statistical information for disability programs and policies. New York: United Nations.

UNITED NATIONS (2003). The collection and dissemination of statistics on disability at the United Nations Statistics Division: Proposals for the future. New York: The Social and Housing Statistics Section, United Nations Statistics Division.

Verdugo Alonso, M.A. (dir) (1995). Personas con discapacidad: perspectivas psicopedagógicas y rehabilitadoras. Siglo XXI España Editores.

Verdugo Alonso, M.A. y ARIAs Martínez, B. (1991) Evaluación y modificación de las actitudes hacia los minusválidos. Revista de Psicología General y Aplicada, 1991, 44 (1), 95-102.

WEISEL, A. AND FLORIAN, V. (1990). Same- and cross-gender attitudes toward persons with physical disabilities. Rehabilitation Psychology, 35(4), 229-238.

White, K.M.; Robinson, N.G.; Young, R.McD.; Anderson, P.J.; Hyde, M.K.; GreenbanK, S.; Rolfe, T.; KeANE, J.; VARDON, P.; BASKeRVILle, D. (2008). Testing an extended theory of planned behaviour to predict young people's sun safety in a high risk area. British Journal of Health Psychology, 13,3, 435-448. 